\title{
A case of hiccoughs
}

\author{
Umasuthan Srirangalingam, Radha Selvaratnam, John P Monson and Ashley B Grossman
}

\section{Case presentation}

An elderly Asian man returned from Bangladesh with an 18month history of intermittent hiccoughs, anorexia, weight loss and fever. He had type 2 diabetes on insulin therapy with deteriorating glycaemic control. Examination revealed a thin, pale man, with intermittent hiccoughs, darkening of palmar creases and mucous membranes, angular stomatitis, oral ulceration and mild epigastric tenderness. He appeared clinically dry. He was noted to have a spiking fever to $38.5^{\circ} \mathrm{C}$ and became notably hypotensive with a blood pressure $82 / 35 \mathrm{~mm} / \mathrm{Hg}$ during his admission. Initial investigations are shown in Table 1. Chest radiography was unremarkable and an abdominal ultrasound noted a $13.5 \mathrm{~cm}$ splenomegaly. There was no evidence for a subphrenic abscess.

\section{Differential diagnosis}

This subject's initial presentation was relatively non-specific but in association with hypotension would fit with a diagnosis of Addisonian crisis. An infective process, particular tuberculosis (TB), would be high on the differential list given the patient's intermittent fever. Hiccoughs were a persistent feature of the initial presentation and focused attention on ruling out abdominal pathology, particular a subphrenic collection and uraemia. Examination and initial investigation did not identify evidence of a neurological or respiratory cause for hiccoughs. Both hyponatraemia and hyperglycaemia have been associated with persistent hiccoughs.

\section{Initial management}

The patient was treated symptomatically on initial presentation. Antipyretic medication was administered for the fever. Fluid resuscitation with normal saline was instigated when the patient became hypotensive. Blood sugars were monitored more intensively and his insulin titrated appropriately.

\section{Case progression}

Given the history, a serum cortisol was taken at 09.00. This revealed a cortisol measurement of $<50 \mathrm{nmol} / \mathrm{l}$. A subsequent

Umasuthan Srirangalingam, ${ }^{1}$ specialist registrar in endocrinology;

Radha Selvaratnam, ${ }^{2}$ specialist registrar in acute medicine;

John P Monson, ${ }^{3}$ emeritus professor of clinical pharmacology;

Ashley B Grossman ${ }^{1}$ professor of neuroendocrinology

${ }^{1}$ St Bartholomew's Hospital, London; ${ }^{2}$ East Surrey Hospital, Redhill,

Surrey; ${ }^{3}$ The London Clinic, London short synacthen test demonstrated no response to adrenorcorticotropic hormone (ACTH) $(0 \mathrm{~min}<50 \mathrm{nmol} / \mathrm{l}, 30 \mathrm{~min}$ $52 \mathrm{nmol} / \mathrm{l}, 60 \mathrm{~min} 50 \mathrm{nmol} / \mathrm{l})$. Plasma ACTH was unequivocally raised (340 ng/l, normal 10-80) confirming primary adrenal failure. An abdominal computed tomography (CT) scan identified bilateral diffuse adrenal enlargement without calcification (Fig 1a).

Further investigations revealed a serum 17-OH-progesterone was not raised, ruling out a diagnosis of congenital adrenal hyperplasia (CAH). Serum aldosterone was low, as expected with primary adrenal failure, but plasma renin levels were also undetectable. Plasma and urinary catecholamines were within the normal range. A Heaf test was negative. A CTguided biopsy of the adrenals was carried out. This revealed the presence of numerous small yeast-like structures on polarisation microscopy which were strongly positive with Grocott's methenamine silver stain, suggesting a diagnosis of Histoplasma capsulatum (Fig 1b). This was confirmed by serology and culture.

The patient was commenced on parenteral glucocorticoid (hydrocortisone) treatment initially and then converted to oral replacement. His symptoms significantly improved and the pyrexia was rapidly abolished. Mineralocorticoid (fludrocortisone) replacement therapy was subsequently commenced. Histoplasmosis was treated initially with ambisone (lysosomal amphotericin-B) intravenously and then oral itraconazole. His general condition improved, and he was discharged home.

A final diagnosis of Addison's disease with bilateral adrenal enlargement secondary to disseminated histoplasmosis was made.

Table 1. Initial laboratory investigations.

$\begin{array}{lll} & \text { Values } & \text { Normal range } \\ \text { Haemoglobin } & 12 \mathrm{~g} / \mathrm{dl} & 13.5-17.5 \times 10^{9} / \mathrm{I} \\ \text { White cell count } & 7.2 \times 10^{9} / \mathrm{l} & 4-11 \times 10^{9} / \mathrm{I} \\ \text { Eosinophils } & 1.3 \times 10^{9} / \mathrm{I} & 0.02-0.5 \times 10^{9} / \mathrm{I} \\ & & \\ \text { Sodium }(\mathrm{mm}) & 127 & 135-145 \\ \text { Potassium }(\mathrm{mm}) & 4.2 & 3.5-5.0 \\ \text { Creatinine }(\mu \mathrm{m}) & 114 & 60-90 \\ \text { Bicarbonate }(\mathrm{mm}) & 21 & 22-28 \\ \text { Corrected calcium }(\mathrm{mm}) & 2.69 & 2.1-2.6 \\ \text { Albumin }(\mathrm{g} / \mathrm{l}) & 31 & 40-55 \\ \text { Glucose }(\mathrm{mm}) & 22.5 & 4-6 \text { (fasting) } \\ \text { C-reactive protein }(\mathrm{mg} / \mathrm{l}) & 23 & <10\end{array}$




\section{Discussion}

Though rare, bilateral adrenal enlargement has an important differential diagnosis. Differentials include tumours such as lymphoma, bilateral adrenocortical adenomas or phaeochromocytomas. Metastasis of tumours to the adrenal, adrenal haemorrhage (including the Waterhouse-Friderichsen syndrome) and congenital adrenal hyperplasia may all present with bilateral adrenal enlargement. Infections, most commonly TB but also other mycoses, are also important in the differential diagnosis.

Early in the initial assessment for adrenal enlargement several important issues must be clarified. These include the cortisol status of the subject and the need for glucocorticoid replacement, and evidence for a phaeochromocytoma, catecholamine excess and the need for sympathetic blockade. An adrenal biopsy may be necessary to secure the diagnosis and is indicated in the context of Addison's disease and adrenal enlargement.
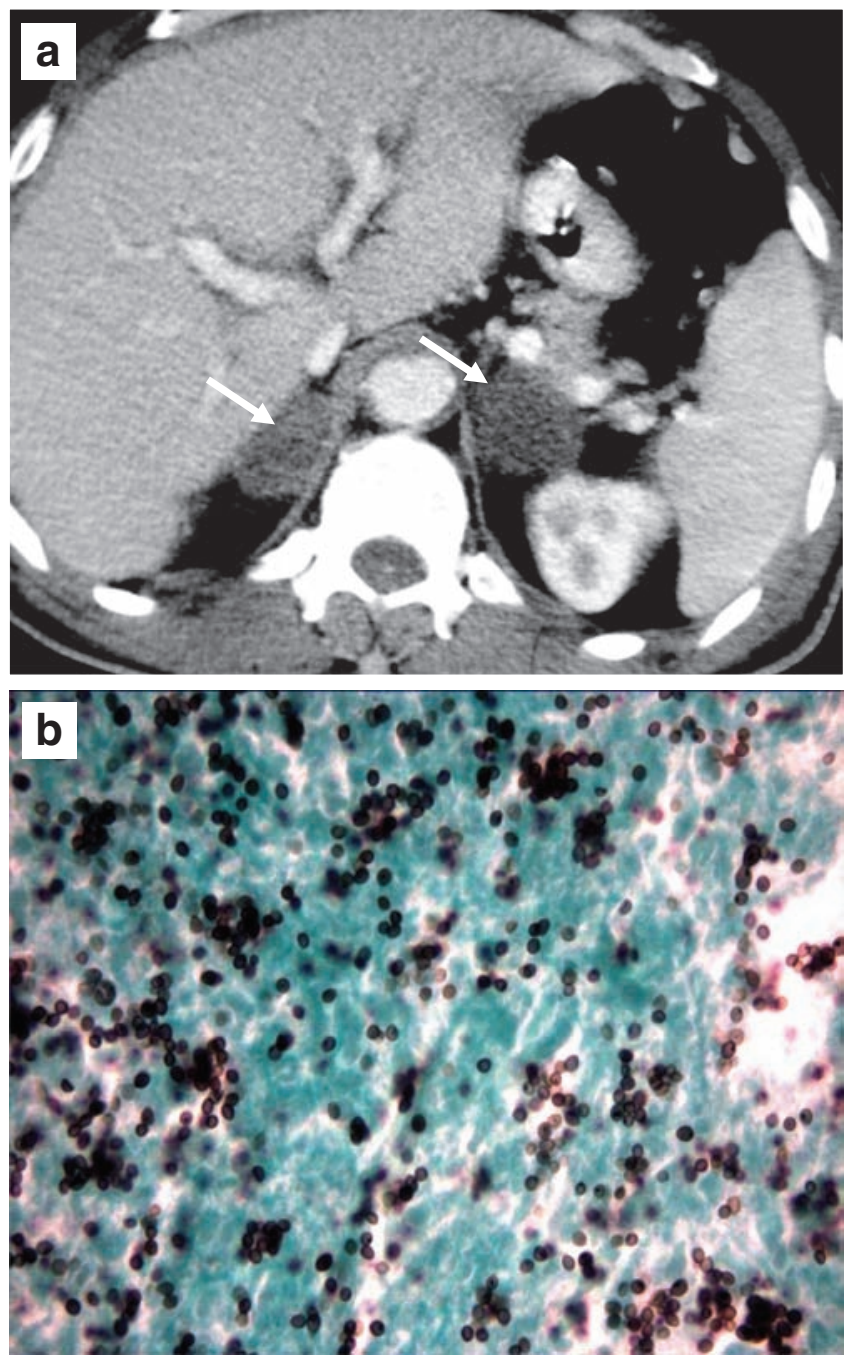

Fig 1. (a) Computed tomography (CT) abdomen (axial) demonstrates bilateral adrenal enlargement (white arrows). (b) Histology from CT-guided biopsy sample with Grocott stain revealing infiltration of histoplasma.
Histoplasma capsulatum, a dimorphic fungus, is the most common endemic mycosis worldwide. Found in the soil of bat and avian habitats, transmission occurs via aerolised spores. Common in North America (Ohio and Mississippi valleys), Latin America and East Africa, in endemic regions over $80 \%$ of the population may have been infected. ${ }^{1}$ Immunocompromised subjects are at greater risk. Acute infection may resemble a flu-like illness but many subjects remain asymptomatic. This case illustrates a classical presentation of disseminated histoplasmosis with probable hepatosplenic and confirmed adrenal involvement, mouth ulcers, fever and weight loss. Other features include lymphadenopathy, meningitis and diffuse interstitial lung infiltrates. Adrenal involvement is thought to occur in up to $10 \%$ of cases but histoplasmosis as a cause of adrenal failure is rare $(<1 \%)$, with $\mathrm{TB}$ being the most common infective cause $(20 \%))^{2,3}$ As such, there is a justifiably low threshold for considering anti-tuberculous therapy in patients with adrenal failure. However, similarities in presentation and a high mortality associated with untreated disseminated histoplasmosis (between $80 \%$ and $100 \%$ ) make it an important diagnosis to consider despite its relative rarity, especially in patients coming from endemic areas. ${ }^{4}$ Laboratory diagnostics included antigen detection, serology and culture. Haematoxylin and eosin $(\mathrm{H} \& \mathrm{E})$ staining with polarised light demonstrates the typical morphology of small yeast-like structures in addition to strong positivity with Grocott staining (Fig 1b). Treatment for severe acute or disseminated histoplasmosis includes intravenous amphotercin-B followed by oral itraconazole or ketoconazole which may require a prolonged course of treatment, up to one year.

Addisonian crisis is a medical emergency requiring a combination of fluid resuscitation, glucose and glucocorticoid administration. A new diagnosis may be secured by demonstrating an inappropriately low random cortisol and elevated paired ACTH level in the context of acute illness. A short synacthen test may be needed to clarify the diagnosis with equivocal cortisol measurements. This subject demonstrated an Addisonian biochemical picture of hyponatraemia, a metabolic acidosis and mild hypercalcaemia. Fever is a noted feature of adrenal failure. Hiccoughs have not been previously associated with a diagnosis of Addison's disease. Primary adrenal failure would normally be associated with low aldosterone and raised renin levels. In this subject, renin levels where undetectable suggesting an additional diagnosis of hyporeninaemic hypoaldosteronism (type IV renal tubular acidosis), likely to be a consequence of renal damage or autonomic dysfunction secondary to long-standing diabetes.

While the case presented here describes a rare cause of bilateral adrenal enlargement, it highlights the need to be aware of the possible differential. Glucocorticoid and catecholamine status must be actively addressed and TB considered high on the differential list. For this particular subject, treatment resulted in resolution of the hiccoughs. 


\section{References}

1 Edwards LB, Acquaviva FA, Livesay VT et al. An atlas of sensitivity to tuberculin, PPD-B, and histoplasmin in the United States. Am Rev Respir Dis 1969;99:1-132.

2 Mukherjee JJ, Villa ML, Tan L et al. Bilateral adrenal masses due to histoplasmosis. J Clin Endocrinol Metab 2005;90:6725-6.

3 Goodwin RA, Jr., Shapiro JL, Thurman GH et al. Disseminated histoplasmosis: clinical and pathologic correlations. Medicine (Baltimore) 1980;59:1-33.
4 Sarosi GA, Voth DW, Dahl BA et al. Disseminated histoplasmosis: results of long-term follow-up. A center for disease control cooperative mycoses study. Ann Intern Med 1971;75:511-6.

Address for correspondence: Dr U Srirangalingam, Department of Endocrinology, St Bartholomew's Hospital, West Smithfield, London EC1M 7BE.

Email: u.srirangalingam@qmul.ac.uk

\title{
Acute renal failure in diabetes: looking beyond diabetic retinopathy
}

\author{
Piya Sen Gupta, Megan Crofts, Kate Antrobus and John V Anderson
}

\section{Case presentation}

A 43-year-old construction worker was admitted to the emergency department with increasingly severe right-sided abdominal pain of three days' duration. There had been no precipitant injury and it had not improved with regular ibuprofen. He had type 1 diabetes of 20 years' duration treated with once daily basal glargine and mealtime NovoRapid ${ }^{\circledR}$ insulin. Other medications were simvastatin $80 \mathrm{mg}$ od, losartan $25 \mathrm{mg}$ od and perindopril $4 \mathrm{mg}$ od. His complications of diabetes included bilateral photocoagulation-treated retinopathy but no known peripheral neuropathy or nephropathy. He also had hypertension, smoked 10 cigarettes daily and had a pleurodesis procedure following a pleural drain for a parapneumonic effusion five years previously.

Examination revealed an alert and lucid (Glasgow Coma Score (GCS) 15/15) Causasian man with evidence of hypovolaemia (dry mucous membranes), cool peripheries, temperature $36.0^{\circ} \mathrm{C}$, pulse $86 / \mathrm{min}$, blood pressure $139 / 87 \mathrm{mmHg}$ and respiratory rate $15 / \mathrm{min}$. Cardiac examination was normal but chest examination revealed dullness to percussion and reduced breath sounds at the left base. He had a distended abdomen with right flank tenderness. Baseline admission investigations are shown in Box 1.

Piya Sen Gupta, diabetes registrar; Megan Crofts, core medical trainee 2; Kate Antrobus, foundation year 2; John V Anderson, consultant diabetologist

Department of Diabetes, Homerton University Hospital, London

\section{What is the differential diagnosis and the most likely diagnosis?}

His main problems are abdominal pain and renal failure on a background of chronic type 1 diabetes. With no known previous nephropathy, it is safest to assume this is acute kidney injury (AKI), the causes of which can be divided into:

- pre-renal or volume responsive

- intrinsic renal

- post-renal subcategories. $^{1}$

The likelihood of each differential within these divisions is influenced by the specifics of this case.

With his type 1 diabetes, electrolyte abnormalities and abdominal pain as a main presenting feature, diabetic ketoacidosis

\section{Key learning points}

- Acute kidney injury (AKI) requires prompt and careful identification of the underlying cause.

- The principles of AKI management begin with the cessation of further renal insult; careful assessment of volume status is also important.

- Rapidly progressive intrinsic renal disease is rare but it is important to diagnose so that directed therapy can follow.

- Although patients with longstanding diabetes can have chronic kidney disease, 'diabetic nephropathy' as the cause of renal failure should never be assumed without confirmation.

- Renal function decline in diabetes warrants urgent establishment of whether this is a new phenomenon by direct patient questioning and checking hospital and primary care records. 